\title{
Directed Acyclic Graph
}

National Cancer Institute

\section{Source}

National Cancer Institute. Directed Acyclic Graph. NCI Thesaurus. Code C45803.

A graph whose edges are ordered pairs of vertices or nodes and which contains no path that starts and ends at the same vertex. 